\title{
HIGH POWER OPERATION OF THE JLAB IR FEL DRIVER ACCELERATOR*
}

\author{
S. Benson", K. Beard, G. Biallas, J. Boyce, D. Bullard, J. Coleman, D. Douglas, F. Dylla, R. Evans, \\ P. Evtushenko, C. Hernandez-Garcia, A. Grippo, C. Gould, J. Gubeli, D. Hardy, C. Hovater, \\ K. Jordan, M. Klopf, R. Li, W. Moore, G. Neil, M. Poelker, T. Powers, J. Preble, R. Rimmer, \\ D. Sexton, M. Shinn, C. Tennant, R. Walker, G. Williams, and S. Zhang \\ TJNAF, Newport News, VA 23606, U.S.A.
}

\begin{abstract}
Operation of the JLab IR Upgrade FEL at CW powers in excess of $10 \mathrm{~kW}$ requires sustained production of high electron beam powers by the driver ERL. This in turn demands attention to numerous issues and effects, including: cathode lifetime; control of beamline and RF system vacuum during high current operation; longitudinal space charge; longitudinal and transverse matching of irregular/large volume phase space distributions; halo management; management of remnant dispersive effects; resistive wall, wake-field, and RF heating of beam vacuum chambers; the beam break up instability; the impact of coherent synchrotron radiation (both on beam quality and the performance of laser optics); magnetic component stability and reproducibility; and RF stability and reproducibility. We discuss our experience with these issues and describe the modus vivendi that has evolved during prolonged high current, high power beam and laser operation.
\end{abstract}

\section{INTRODUCTION}

Though energy recovering linac (ERL) based freeelectron lasers (FELs) are quite complicated devices, operation at very high power requires two things-high average electron beam power (current times voltage) and good laser efficiency at this circulating power level. The IR Upgrade FEL at Jefferson Lab first operated at high power at a wavelength of $5.75 \mu \mathrm{m}$ in the summer of 2004 . The accelerator could deliver $8 \mathrm{~mA}$ of recirculated beam current at $165 \mathrm{MeV}$ (1.33 MW). Using an optical klystron, the efficiency of the FEL was as high as $1.7 \%$ when the accelerator was run at low duty cycle, implying a power limit of $22 \mathrm{~kW}$. We found, however that the laser efficiency was less than $1.1 \%$ when operated $\mathrm{CW}$ at $>8 \mathrm{~kW}$. We were able to operate with 1 second pulses and achieve slightly higher efficiency $(1.2 \%)$, attaining a power of $10.5 \mathrm{~kW}$ during the pulses [1]. The $1.1 \%$ efficiency was achieved with approximately $0.77 \mathrm{MW}$ of beam power. The efficiency at this power was inversely proportional to the electron beam power, resulting in the power being clamped at $\sim 8 \mathrm{~kW}$.

In the spring and summer of 2006 we operated the FEL

\footnotetext{
* This work supported by the Office of Naval Research, the Joint Technology Office, the Army Night Vision Laboratory, the Air Force Research Laboratory, the Commonwealth of Virginia, and by DOE Contract DE-AC05-060R23177

"felman@jlab.org
}

with a new permanent magnet wiggler with higher gain but similar efficiency to the previous device. A vacuum accident had reduced the maximum accelerator energy to $120 \mathrm{MeV}$. We typically operated at $115 \mathrm{MeV}$ so that we would have some operational overhead. Operating in the 1 to 3 micron wavelength range, we found that the laser power was clamped at even lower levels than before. The cause of this clamping was found to be mirror distortion due to heating. Using a cryogenically cooled sapphire output coupler we were able to essentially eliminate this distortion and reached a power output of $14.3 \mathrm{~kW} \mathrm{CW}$ at 1.6 microns. This paper discusses the issues that had to be dealt with to produce such a result.

\section{LIMITS TO HIGH CURRENT OPERATION}

Operation of an energy recovering linac at high current with a free-electron laser presents many operational challenges. We discuss several of these challenges here.

\section{Beam Loss Due To Halo}

Operation with $8 \mathrm{~mA}$ of beam implies very low losses everywhere. Loss of even $1 \mu \mathrm{A}$ in an uncooled chamber can lead to unacceptable vacuum rise. Upstream of a permanent magnet wiggler we are limited to about 100 $\mathrm{nA}$ of loss before wiggler lifetime becomes a problem. Achieving such a low level of loss requires understanding and control of halo formation. The electron beam also has outlying features in its phase space that contain a significant fraction of the charge. This is highlighted in figure 1 where we show the charge distribution on an OTR viewer at the end of a long drift just before the final bunching chicane. We found that one can define several beamlets in this phase space that can each be matched to the transport. The best strategy for transporting the beam was to calculate the Twiss parameters from rms spot sizes equal to $1 / 6$ of the full width of the beam. When this was done we could reduce losses in most of the accelerator to less than $100 \mathrm{nA}$ and less than $10 \mathrm{nA}$ at the wiggler.

Beam loss between the gun and pre-accelerator had to be extremely low to preserve the cathode lifetime. The demonstrated lifetime was on the order of $500 \mathrm{C}$ independent of current ( $>16$ hours of $8 \mathrm{~mA}$ operation)

\section{Vacuum Rise From Other Sources}

We often see vacuum pressure rises downstream of dipole magnets that are not associated with beam loss. We believe that these pressure rises are due to emission of 
coherent synchrotron radiation (CSR) at points where the electron bunches are short. This beam is capable of emitting hundreds of Watts of $\mathrm{THz}$ radiation in this manner [2]. The same beam conditions that produce large amounts of CSR are also ideal for generation of wake fields and resistive wall heating. All these sources can be managed by careful design of the vacuum chamber to keep transitions in chamber cross-section to a minimum absorb wake fields and CSR in water cooled loads, and cool any narrow chambers to manage resistive wall heating. Presently we can run $8 \mathrm{~mA}$ for up to one hour before vacuum rise due to uncooled elements forces us to temporarily shut off the beam. Note that this situation must be improved by at least two orders of magnitude before an ERL based synchrotron light source can be operated.

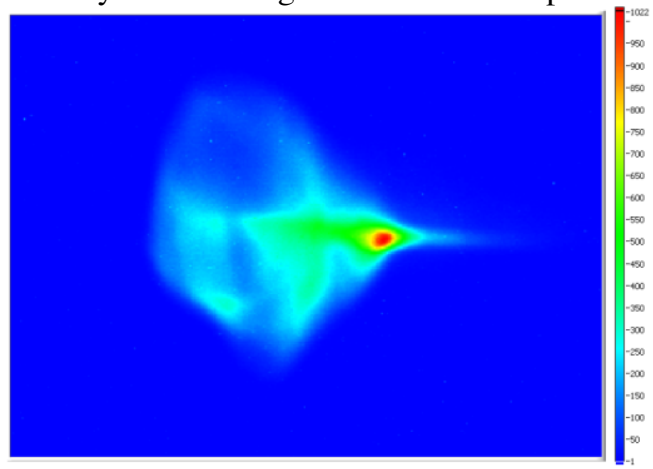

Figure 1: OTR image of the beam after a long drift showing complicated phase space distribution.

Finally, RF window heating can lead to vacuum rises in the waveguides delivering RF to the SRF cavities. This is a major limitation for the IR upgrade. The maximum power with which we could drive the injector cavities was approximately $40 \mathrm{~kW}$. This limited the current to about 8 $\mathrm{mA}$. A new window design should allow higher current.

\section{Energy Recovery Challenges}

Beam can also be lost due to the momentum distribution exceeding the energy recovery system acceptance. One must set up the system to have as large a momentum aperture as possible, preferably limited only by the vacuum chamber aperture. So-called "incomplete" energy recovery must be used if the accelerated beam is close to crest but the energy acceptance is large. The mean decelerating phase offset from trough $\phi_{d e c}$ must be at least $\phi_{\text {dec }}=\sqrt{\Delta E / V_{\text {linac }}}$ where $\Delta E$ is the full energy spread and $V_{\text {linac }}$ is the linac voltage. If this phase is greater than the accelerating phase offset, then energy recovery is described as incomplete since the final energy is higher than the injected energy. Once the decelerating phase has been set, the multipoles in the energy recovery arc must be set to match the shape of the decelerating waveform to third order in momentum offset. This will produce a small energy spread at the dump even for an exhaust energy spread of over $12 \%$. The final challenge is to balance the chromatic aberration by properly balancing the telescopes between the FEL wiggler and the linac so that the transverse match to the dump is insensitive to energy offset.

\section{Other High Current Effects}

Since efficiency in the FEL fell off as the CW current was increased we looked at possible degradation in the electron beam quality vs. current. Figure 2 shows the rms phase jitter near the FEL as a function of current. There is no obvious trend that would explain the fall-off in efficiency. The laser efficiency did not go through a minimum at $2.5 \mathrm{~mA}$ but decreased monotonically with current. The laser is also more sensitive to high frequency phase jitter, which is relatively constant and much smaller than the low frequency jitter.

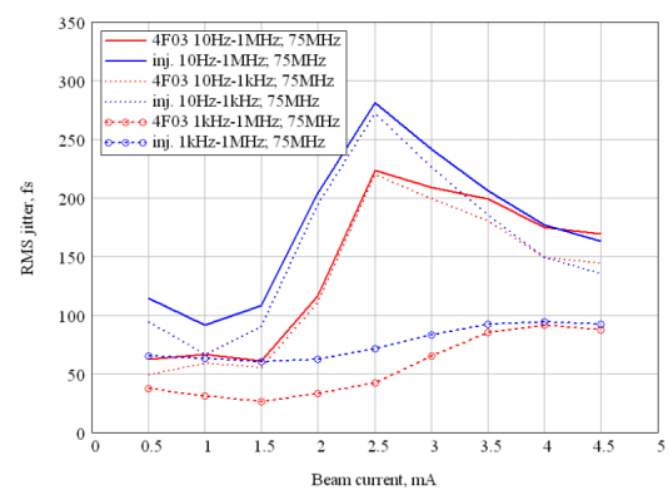

Figure 2: Phase noise as a function of average beam current. Dashed curves are for $1 \mathrm{kHz}$ to $1 \mathrm{MHz}$. Dotted curve are for $10 \mathrm{~Hz}$ to $1 \mathrm{kHz}$. Solid curves are for the full spectrum.

We also looked at transverse position movement and found only a small shift in the average position vs. current. We had to learn to resteer at high current to compensate for this effect. We also saw phase pulling that would change the bunching of the electrons at the wiggler. We found that the CSR induced energy spread could be used as a diagnostic to quickly recover the proper phasing at high current.

We have discussed beam breakup limits in previous work [3]. Using a beam rotator we were able to routinely raise the $\mathrm{BBU}$ threshold to a value much higher than 10 $\mathrm{mA}$. In general then, BBU was not a problem.

\section{LASER EFFICIENCY}

The wiggler used for the experiments noted here had 30 $5.5 \mathrm{~cm}$ periods and had a maximum $\mathrm{rms} \mathrm{K}^{2}$ of 8.4. This allowed us to tune over a very large wavelength range at single electron beam energy. The wiggler was optimized for the $1-2 \mu \mathrm{m}$ wavelength range but lased from 0.65 to $5.2 \mu \mathrm{m}$. The single pass gain was typically over $80 \%$. This allowed us to increase the output coupling and reduce the circulating power in the cavity. We were able to operate with a $20 \%$ output coupler at $1.6 \mu \mathrm{m}$. The laser efficiency with this output coupler was as high as $1.7 \%$.

In order to achieve the high gain and efficiency in the FEL several things had to be done:

- The match to the wiggler had to be optimized so that the gain was high but the beam losses were extremely small. This was difficult to do using simple emittance measurements and matching due 
to irreproducibility in the focusing magnets. We developed techniques that allowed us to reset the focusing lattice using beam-based measurements. This step was also important in minimizing longitudinal emittance growth in the beam during acceleration and transport to the wiggler.

- Dispersion had to be corrected at the wiggler to second order in the energy offset. This had to take into account higher order terms such as the $T_{346}$ term. This means that the dispersion at the wiggler depended on the orbit in the first arc. This step was essential for managing beam loss since it was found that the halo has a large energy spread.

- The longitudinal match to the wiggler had to be set to second order. This produces an upright distribution at the wiggler. In practice we slightly tilted the distribution to greatly reduce the CSR-induced energy spread. Typical rms bunch lengths were $\sim 170$ fsec (peak current $>300 \mathrm{~A}$ ).

- The RF feedback loops had to be tuned so that the phase and energy of the electrons were sufficiently stable so that the gain was independent of time.

When the accelerator was tuned up properly and the optical cavity was well aligned the efficiency at low duty cycle would be between $1.5 \%$ and $1.7 \%$. When the current was raised however the efficiency would fall. This is shown in figure 3.

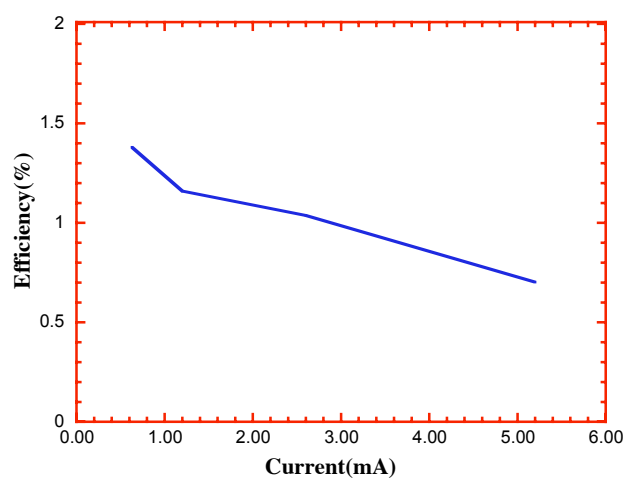

Figure 3: Laser efficiency vs. current for room temperature mirrors. Power at high current is clamped at $4.2 \mathrm{~kW}$.

Though a fall-off in efficiency with current could be due to some degradation in the electron beam quality with current, we could see no evidence for such degradation. We did, however, see evidence for mirror heating sufficient to affect the efficiency. FEL simulations have indicated that wavefront distortions due to mirror heating cannot exceed $1 / 5$ of a wave [4]. The power that a mirror can absorb before attaining this much wavefront distortion is only a function of the thermal properties of the mirror [5]. We found that the absorbed power when the power was clamped matched this prediction well and showed the predicted linear dependence on wavelength.

The solution to this power limiting effect was to use a cryogenic output coupler. Cryogenic sapphire can absorb hundreds of watts of power with virtually no thermal distortion due to its thermal properties. The efficiency vs. current with the cryo-mirror is show in figure 4. Though the efficiency differed from day to day, it did not vary appreciably with current. We can conclude from this data that the electron beam quality is not strongly degraded at high current as long as we compensate the steering and phase pulling caused by beam loading.

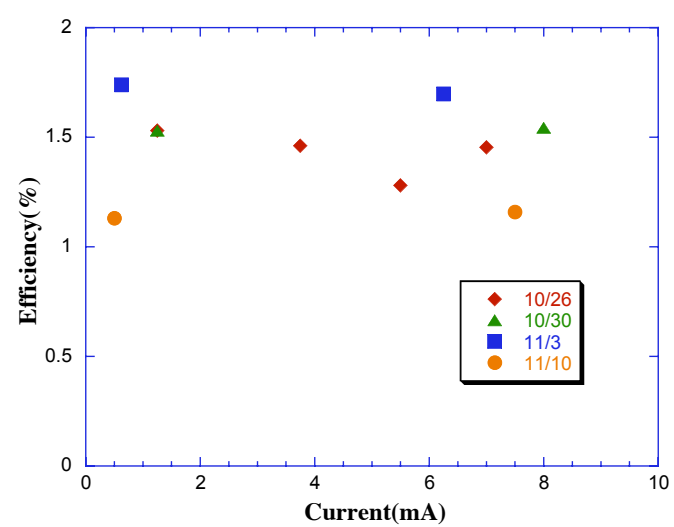

Figure 4: Efficiency of the FEL operating with the cryomirror as a function of current. Data was taken on four different days of operation.

\section{CONCLUSIONS}

Delivery of a high quality, high current beam to a freeelectron laser in an energy recovery linac is a challenging task but we have found no fundamental limits to such delivery. The limitations on the laser efficiency seemed to be due completely to thermal distortion in the mirrors. Cryo-mirrors eliminate distortion and allowed us to scale the power up by a factor of 3 so far. The power is now limited by the electron beam current, which is limited by $\mathrm{RF}$ window heating in the injector accelerating unit.

\section{REFERENCES}

[1] S. Benson et al., "High Power Lasing in the IR Upgrade FEL at Jefferson Lab", Proceedings of the 2004 FEL Conference, Trieste, Italy, 229 (2004).

[2] J. M. Klopf, A. Greer, J. Gubeli, G. R. Neil, M. Shinn, T. Siggins, D. Waldman, G. P. Williams, A. Todd, V. Christina and O. Chubar, "The Jefferson Lab High Power THz User Facility", Nucl. Instrum. Methods A, accepted for publication, (2007).

[3] C. Tennant et al., "Experimental Investigation of Beam Breakup in the Jefferson Laboratory $10 \mathrm{~kW}$ FEL Upgrade Driver", Proceedings of the 2005 PAC conference, Knoxville TN, p. 369.

[4] B. D. McVey, J. C. Goldstein, R. D. McFarland, and B. E. Newnam, SPIE 1441, Laser induced damage in optical materials, H. E. Bennett, L. L. Chase, A. H. Guenther, and B. E. Newnam, Editors, (1991) 447.

[5] S. V. Benson, "What Have We Learned From the Kilowatt IR-FEL at Jefferson Lab?" NIM A483, 1 (2001). 